\title{
Research on an Object Oriented Change Detection Algorithm
}

\author{
Dehui Zhang, 2, a, Yong YANG ${ }^{1, b^{*}}$, Kai SONG ${ }^{2, c}$, Deyu ZHANG ${ }^{2, d}$ \\ ${ }^{1}$ School of Information and Electrical Engineering, Shenyang Agricultural University, Shenyang, \\ 110161, China \\ ${ }^{2}$ School of Information Science and Engineering, Shenyang Ligong University, Shenyang, 110159, \\ China \\ azdh_syit@163.com, byy_syau@163.com, 'songkai_syit@163.com, dzhang_de_yu@163.com
}

\begin{abstract}
Keywords: Objected oriented, Change detection, Multi-temporal, Image segmentation, Similarity Abstract An object oriented change detection algorithm for multi-temporal remote sensing image is proposed in this study. Bi-temporal remote sensing images are firstly processed by multi-scale segmentation. Then similarity between the target object and its neighboring object is calculated by using a formula of similarity calculation. Optimal threshold is selected by using $\mathrm{K}$ means clustering method to estimate whether the two images has similarity. Finally change between the target object and its neighboring object is determined by comparison of similarity and threshold. Our experimental results demonstrate that the proposed algorithm can realize change detection of multi-temporal remote sensing image with high accuracy.
\end{abstract}

\section{Introduction}

With the development of remote sensing technology, massive amounts of remote sensing images have been accumulated [1]. Investigation of how to take advantages of these remote sensing data efficiently and translate them into more valuable knowledge will provide abundant and helpful auxiliary information for relevant departments to make quick and accurate decisions, which also promotes emergence and development of change detection technique based on multi-temporal remote sensing images.

Change detection of multi-temporal remote sensing image is a technique that analyzes multi-temporal high resolution satellite remote sensing images in the same area. It can effectively and quickly detect change of the investigated area by using image processing method. If there is change, then the features and reasons of the change will be interpreted further to realize the analysis and understanding of remote sensing images [2,3].

Many techniques have been developed for traditional change detection, including algebraic method, classification method, model method, time trajectory method, etc [4]. Traditional change detection techniques are based on spectral characteristics of pixels and simply focus on local data, so it cannot effectively analyze and utilize more detailed information [5]. To overcome the limitation of traditional change detection methods, by taking advantages of satellite remote sensing image, we propose a novel object oriented change detection algorithm.

\section{Flowchart of the Algorithm}

The flowchart of the object oriented change detection for remote sensing image is shown in Fig. 1. 


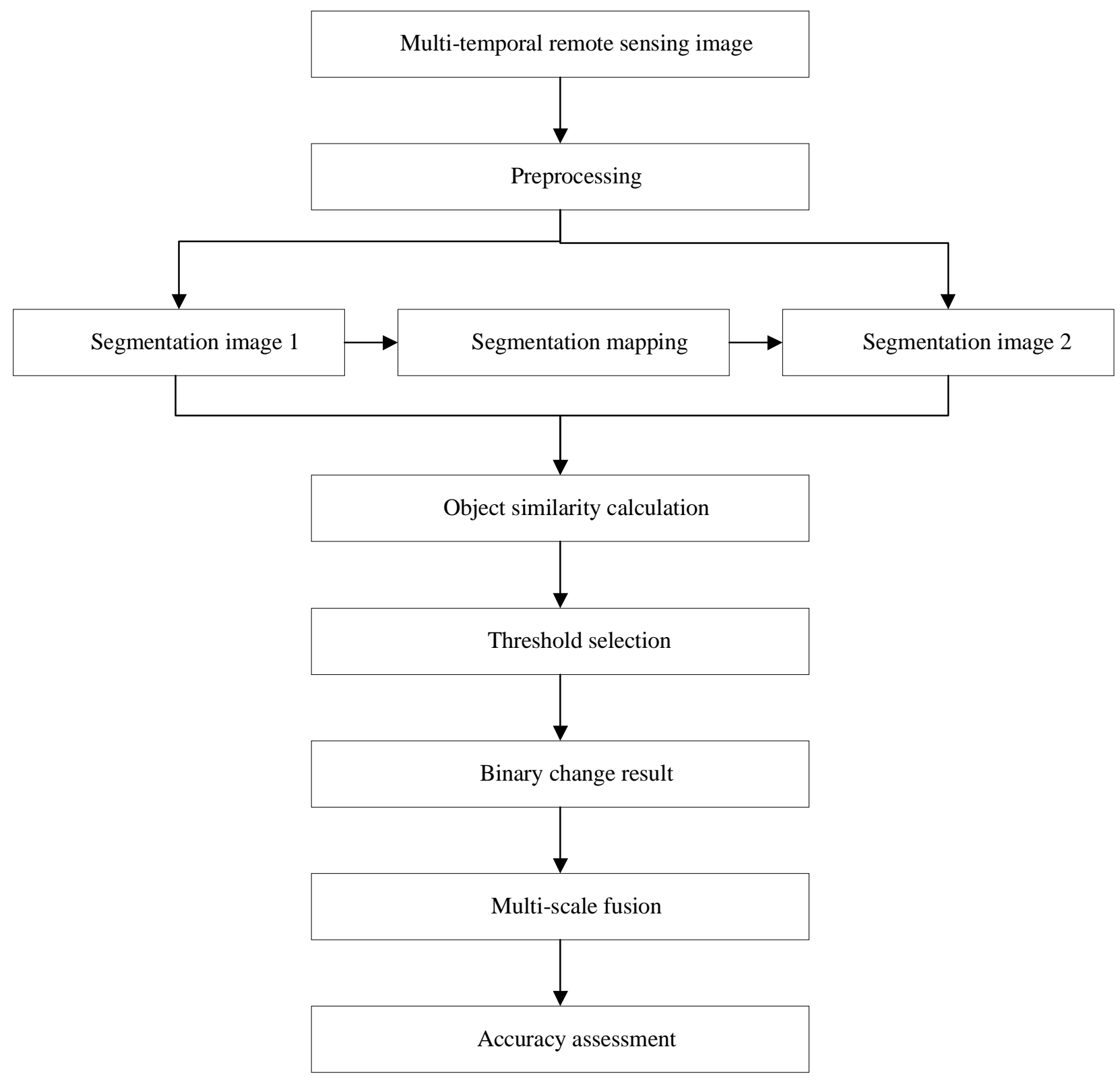

\section{Implementation of Algorithm}

Fig. 1 Flowchart of change detection

\section{Segmentation of Multi-temporal Remote Sensing Image}

Suppose there are two remote sensing images, i.e. remote sensing image 1 and remote sensing image 2, which are taken from the same area at different time. The segmentation of the multi-temporal remote sensing image is as the following:

(1) Remote sensing image 1 was segmented to achieve segmentation image 1 , which was also used as the reference image.

(2) Mapping the segmentation image 1 to the remote sensing image 2 to obtain segmentation image 2. Thus each image object in the segmentation image 1 and the segmentation image 2 includes the same pixel.

\section{Calculation of Similarity between Target Object and Neighboring Object}

(1) Spatial correlation of change area between target object and neighboring object

The segmentation image 1 is used as reference image, so the image object in segmentation image 1 is called "target object", while the image object in segmentation 2 is called "neighboring object" accordingly. When target earth objects change, there is a spatial correlation between image object and changed area. Altogether, there are six relations between the target object, neighboring object and 
changed area, which are shown in Fig. 2. In Fig. 2, thick line represents for target object, thin line for neighboring object and grey shadow for changed area.

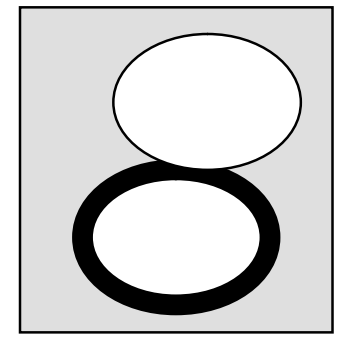

(1)

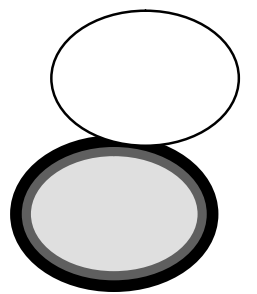

(4)

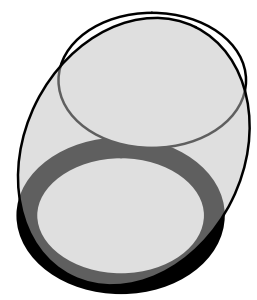

( 2 )

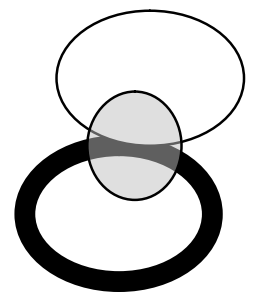

( 5 )

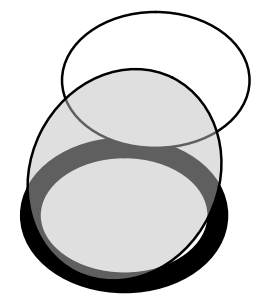

( 3 )

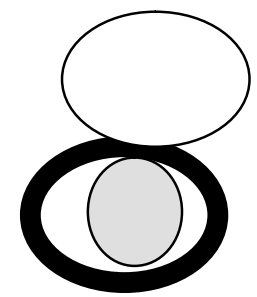

(6)

Fig. 2 Spatial relationship between image object and changed area

Due to the six relationships mentioned above, change of spatial relations will exert an effect on spatial features of multi-temporal object.

a) When changed area is larger than target object and its neighboring object, contrast between target object and its neighboring object decreases;

b) When changed area includes target object and its neighboring object, contrast between target object and its neighboring object decreases;

c) When changed area includes target object and a part of its neighboring object, contrast between target object and its neighboring object decreases;

d) When changed area includes target object, contrast between target object and its neighboring object changes toward unknowing directions, either decreasing or increasing.

e) When changed area lies between target object and its neighboring object, contrast between target object and its neighboring object decreases;

f) When changed area is inside of target object, the internal standard deviation of target object increases.

From the above, we conclude that spatial features of multi-temporal image will be affected under the following 3 conditions:

a) Contrast between target object and its neighboring object decreases;

b) Contrast between target object and its neighboring object changes toward unknowing directions, either decreasing or increasing;

c) Internal standard deviation of target object increases

The spatial features of multi-temporal object is associated with contrast, contrast ratio at different temporal phase and internal standard deviation, so the formula for calculation of similarity between target object and its neighboring object depends on these three parameters.

(2) Formula for calculation of similarity between target object and its neighboring object

When $0<F_{R C i}<1$,

$$
F_{i}=\frac{F_{R C i} \cdot \sum_{\forall(p, q) \in O N_{i}} F_{C p}}{F_{S D i}} .
$$


Otherwise $F_{R C i}>1$,

$$
F_{i}=\frac{\sum_{\forall(p, q) \in O N_{i}} F_{C p q}}{F_{R C i} \cdot F_{S D i}} .
$$

In the formula, $F_{C p q}$ represents for contrast between target object and its neighboring target. $F_{R C i}$ represents for ratio of contrast at different temporal phase. $F_{S D i}$ represents for internal standard deviation.

\section{Threshold Selection}

After similarity between target and its neighboring target is calculated, we need to select appropriate threshold to perform binary segmentation for similarity features. Threshold selection is achieved primarily by using K-means clustering method, which makes the sum of the squared distance of every pixel to their assigned cluster centers minimal [6].

\section{Multi-scale fusion}

For a certain pixel, change detections under different segmentation scales are performed. If times of change are larger than threshold, the pixel will be placed in the fusion result. If times of change are smaller than threshold, the pixel will not be placed in the fusion result.

In our experiment, the multi-temporal high resolution satellite remote sensing images are from multispectral data taken by IKONOS satellite. Remote sensing image 1 was taken in March, 2007 as shown in Fig. 3. Remote sensing image 2 was taken in June, 2010 as shown in Fig. 4.

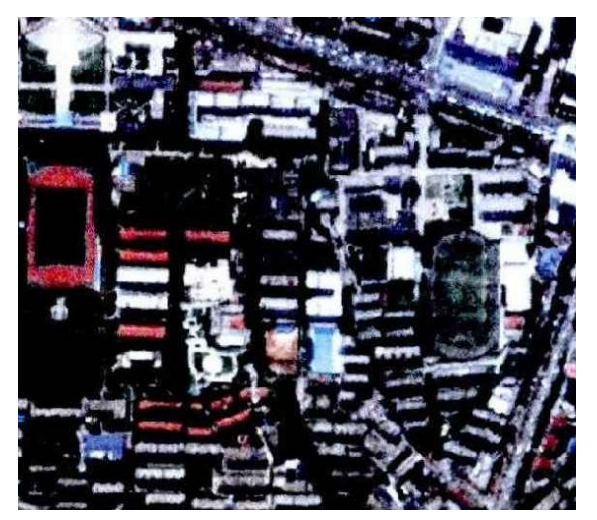

Fig. 3 Remote sensing image 1

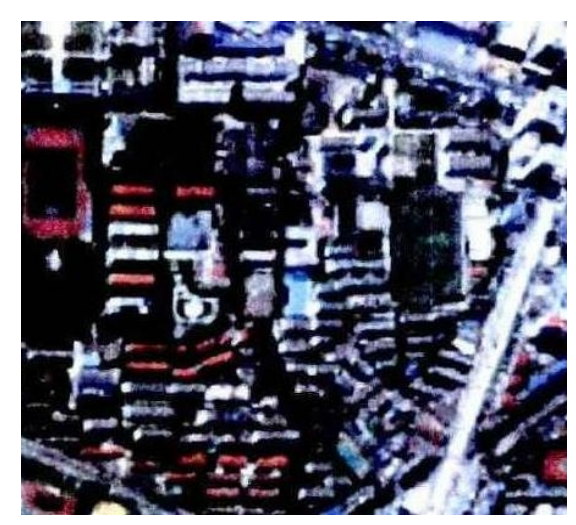

Fig. 4 Remote sensing image 2

Similarity features of object are weighted and combined. Result of change after K-means clustering threshold selection is shown in Fig. 5. To verify the performance of the object oriented change detection algorithm of remote sensing image, reference result of the change detection is obtained by field investigation and visual interpretation (see Fig. 6).

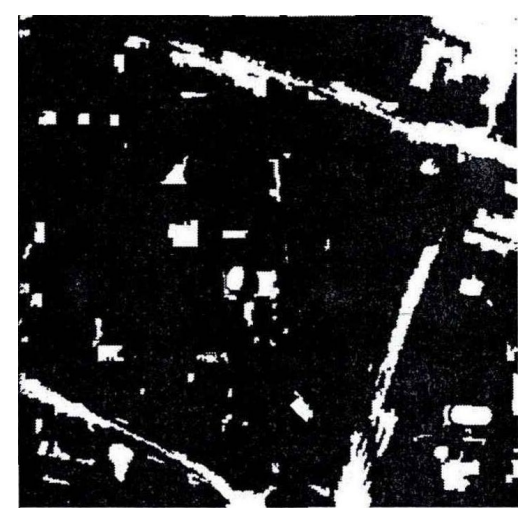

Fig. 5 K-means clustering dectection result

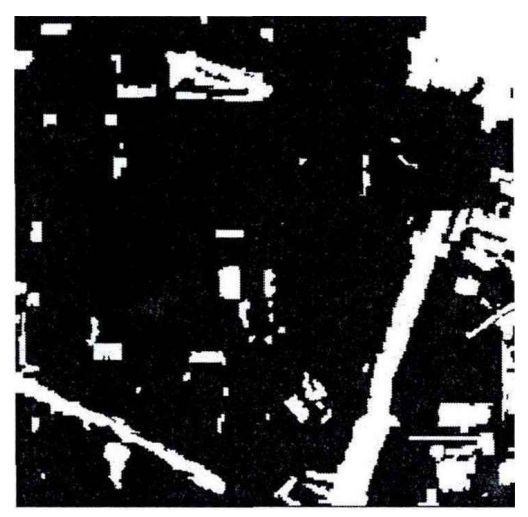

Fig. 6 Reference result

In Fig. 5 and Fig. 6, white represents for changed area and black for unchanged area. The two figures show that detection result obtained by K-means clustering method is consistent with the 
reference result. For multi-temporal change detection of high resolution satellite remote sensing image, level of error of the proposed algorithm is rather low.

\section{Summary}

In this study, we describe the proposal, flowchart and implementation of the algorithm in detail. We also demonstrate by experiment that the proposed object oriented change detection algorithm not only significantly increases change detection efficiency for multi-temporal remote sensing image, but also takes greater advantages of high resolution satellite remote sensing image. Our results might be applied in monitoring of resources, environment (land use and land cover), agriculture (crop growth), and monitoring and evaluation of natural disasters (earthquake, flood, debris flow and forest fires). For change detection of multi-temporal remote sensing image, the development tendency is to increase the detection accuracy by integrating various change detection methods. The change detection results with high accuracy will provide correct and timely help for our decision making.

\section{References}

[1] R. Duan X, Tian Z, Ding M, Zhao W, Registration of remote-sensing images using robust weighted kernel principal component analysis, International Journal of Electronics and Communications, 2013, vol.67, pp. 20-28.

[2] Blaschke T, Hay G J, Kelly M, Lang S, Hofmann P, Addink E, Queiroz Feitosa R, van der Meer F, van der Werff H, van Coillie F, Tiede D, Geographic object-based image analysis-towards a new paradigm, ISPRS Journal of Photogrammetry and Remote Sensing. 87 (2014) 180-191.

[3] Yu C J , Jin B L , Lu Y G , Chen X W , Yi Z M , Zhang K , Wang S. Multi-threshold Image

Segmentation Based on Firefly Algorithm[C]. Ninth International Conference on Intelligent

Information Hiding and Multimedia Signal Processing, 2013, pp.415-419.

[4] Forestier G, Wemmert C, Puissant A. Coastal image interpretation using background knowledge and semantics[J]. Computers \& Geosciences, 54 (2013) 88-96.

[5] Kharol, S.K. , Kaskaoutis, D. , Badarinath, K. , et al. Influence of land use/land cover (LULC)

changes on atmospheric dynamics over the arid region of Rajasthan state, India. Journal of Arid Environments, 88 (2013) 90-101.

[6] Verburg PH , Neumann K , Nol L . Challenges in using land use and land cover data for global change studies. Global Change Biology, 17 (2011) 974-989. 